\title{
Stressor interaction networks suggest antibiotic resistance co-opted from stress responses to temperature
}

\author{
Mauricio Cruz-Loya $\mathbb{1}^{1} \cdot$ Tina Manzhu Kang ${ }^{2} \cdot$ Natalie Ann Lozano ${ }^{2} \cdot$ Rina Watanabe $^{2}$ - Elif Tekin ${ }^{1,2}$. \\ Robert Damoiseaux ${ }^{3} \cdot$ Van M. Savage ${ }^{1,2,4} \cdot$ Pamela J. Yeh ${ }^{2,4}$
}

Received: 6 February 2018 / Revised: 12 June 2018 / Accepted: 4 July 2018 / Published online: 31 August 2018

(c) International Society for Microbial Ecology 2018

\begin{abstract}
Environmental factors like temperature, pressure, and $\mathrm{pH}$ partly shaped the evolution of life. As life progressed, new stressors (e.g., poisons and antibiotics) arose as part of an arms race among organisms. Here we ask if cells co-opted existing mechanisms to respond to new stressors, or whether new responses evolved de novo. We use a network-clustering approach based purely on phenotypic growth measurements and interactions among the effects of stressors on population growth. We apply this method to two types of stressors-temperature and antibiotics- to discover the extent to which their cellular responses overlap in Escherichia coli. Our clustering reveals that responses to low and high temperatures are clearly separated, and each is grouped with responses to antibiotics that have similar effects to cold or heat, respectively. As further support, we use a library of transcriptional fluorescent reporters to confirm heat-shock and cold-shock genes are induced by antibiotics. We also show strains evolved at high temperatures are more sensitive to antibiotics that mimic the effects of cold. Taken together, our results strongly suggest that temperature stress responses have been co-opted to deal with antibiotic stress.
\end{abstract}

\section{Introduction}

Organisms encounter and respond to myriad stressors [1,2]. Stresses to bacteria can come in many different forms, such as use of antibiotics [3, 4], changes in temperature [5], variations in salt concentration or $\mathrm{pH}[6]$, or a lack of

These authors contributed equally: Mauricio Cruz-Loya, Tina Manzhu Kang.

Electronic supplementary material The online version of this article (https://doi.org/10.1038/s41396-018-0241-7) contains supplementary material, which is available to authorized users.

Pamela J. Yeh

pamelayeh@ucla.edu

1 Department of Biomathematics, University of California, David Geffen School of Medicine, Los Angeles, CA 90095, USA

2 Department of Ecology and Evolutionary Biology, University of California, Los Angeles, CA 90095, USA

3 Department of Medical and Molecular Pharmacology, University of California, David Geffen School of Medicine, Los Angeles, CA 90095, USA

4 Santa Fe Institute, Santa Fe, NM 87501, USA nutrients [7]. Cellular responses to these stressors vary but can range from specific subcellular mechanisms such as efflux pumps that pump out toxic compounds $[8,9]$ and outer membrane porins that regulate osmolarity [10] to more global modulation that includes dormancy or quiescence under nutrient limitation [11].

Temperature and pressure gradients are stressors that living organisms have needed to contend with since life first evolved [12-15]. Indeed, phylogenetic evidence based on ribosomal RNA sequences places the emergence of hyperthermophiles near the root of the tree of life [16, 17], so sensing, responding, and adapting to pressure and temperature must constitute some of the oldest adaptations in nature. The heat-shock response machinery, which is a mechanism for cells to deal with the noxious effects of high temperatures, is present across all domains of life and is highly conserved [18-21]. In contrast, the first antibiotics are thought to have arisen more recently in evolutionary history, between 2 billion and 40 million years ago [22]. Consequently, it seems likely that adaptive responses to variations in environmental temperature evolved before responses to antibiotics.

It seems possible that some of the mechanisms that confer resistance to variations in temperature have been 
co-opted to deal with antibiotic stress as well, especially since temperature and drugs harm many of the same cellular components. For instance, high temperatures and antibiotics (e.g., macrolides and aminoglycosides) both affect protein synthesis and folding [23, 24]. In addition to functional overlap, there are compelling reasons for cells to evolve a relatively small suite of stress responses to multiple types of stressors. Developing a novel stress response requires investment in terms of genetic material (i.e., information), protein production, time to evolve, and energy to support simultaneous responses. Thus, it is inefficient for a cell or organism to evolve an independent response for every single stressor it encounters. Greater efficiency can be achieved if cells can co-opt similar pathways to respond to different stressors [25-27]. A prime example of this evolutionary strategy in bacteria is the alternative sigma factor $\sigma^{\mathrm{S}}$, which regulates the expression of $>70$ genes that confer resistance against stresses as diverse as temperature change, starvation, $\mathrm{pH}$, and DNA damage [28-30].

It is natural to ask which response mechanisms evolved first, whether these original responses were co-opted to respond to other stressors, and how much overlap exists among how stressors affect bacteria. It has previously been shown that heat-shock proteins are induced by some antibiotics [31], and that resistance to antibiotics can be temperature-dependent [32]. Furthermore, selection of heatresistant Escherichia coli results in the evolution of resistance to rifampicin, despite the drug being absent during the selection process [33]. Additionally, overexpression of heatshock proteins increases short-term survival of bacteria exposed to aminoglycosides [34]. Other stress responses, such as those for nutrient starvation and oxidative stress, have also been linked to the emergence of antibiotic resistance [35].

Despite these intriguing, isolated subcellular studies, we are unaware of any systematic, comprehensive study of these overlaps and co-opting. Typically, the overlap between cellular responses to stress has been studied by isolating subcellular parts and attempting to piece together the information involved to understand stress responses at a whole-cell level. Here, we take a reverse and complementary approach: by studying the effect of perturbations on the whole system, we gain more insights into the mechanisms of its specific parts. It is now feasible to accomplish this with network-clustering methods that reveal mechanism of action of antibiotics [36, 37]. For these clustering methods, interactions between drugs are inferred based on growth assays of bacteria exposed to antibiotic combinations. Interactions between drugs are typically characterized in one of three ways: additivity (drugs have independent effects on growth), synergy (the drug combination is more potent for inhibiting growth than expected based on their single effects), or antagonism (the drug combination is less potent than expected based on their single effects) [38]. Networks are then constructed in which edges represent these interactions and nodes represent drugs. Because drugs with similar functional effects in the cell tend to have very similar interactions with other drugs, clustering this network according to interaction profiles (a procedure called monochromatic clustering) has been shown to yield groups of drugs with the same mechanisms of action [37]. The interaction profile of a drug can thus reveal its functional effect in the cell.

In this paper, we generalize and extend the ideas of monochromatic clustering. We develop a clustering method for stressor interaction networks (SINs) to categorize nondrug stressors that affect bacterial growth, and we use this novel approach to reveal information about the shared effects of temperature and antibiotics on the cell. To accomplish this, we collect comprehensive data on bacterial growth in the presence of each stressor separately, and when pairs of stressors are present simultaneously. These measurements are used to determine interactions between stressors and construct a SIN. We then find groups of antibiotics that have similar physiological effects to low- and high-temperature stress by grouping stressors that interact similarly with other stressors, as revealed through the SIN clustering analysis. In this way, we systematically analyze overlap between stress responses to temperature and antibiotics to assess the extent that cellular responses to drugs co-opt and mimic the responses to temperature, an ancient stressor.

Here, by systematically carrying out experiments and performing network-clustering analysis, we determine the overlap between the physiological effects of multiple classes of antibiotics and those of six temperatures, ranging from near normal to extreme, in E. coli. Moreover, we confirm that temperature-response genes are involved in responding to antibiotics by measuring genome-wide transcriptional expression with a library of about 1800 strains that contain fusions of green fluorescent protein (GFP) with $E$. coli promoters. Finally, to assess the extent to which adaptation to temperature confers antibiotic resistance, we evaluate the cross-resistance to antibiotics of high-temperature-adapted strains obtained in a previous study [33]. Our results all provide evidence that elements of the low- and hightemperature stress responses have been co-opted through evolution to combat multiple classes of antibiotics.

\section{Materials and methods}

\section{Bacterial strain}

The study used BW25113, a derivative of the F-, $\lambda-, E$. coli K-12 strain BD792 (CGSC6159) [39]. A single colony was inoculated into $2 \mathrm{~mL}$ of $\mathrm{LB}$ media $(10 \mathrm{~g} / \mathrm{L}$ tryptone, $5 \mathrm{~g} / \mathrm{L}$ 
Table 1 Antibiotics and doses used for the antibiotictemperature SIN clustering experiments

\begin{tabular}{|c|c|c|c|c|}
\hline Compound & Abbreviation & Class/cellular target & $\begin{array}{l}\text { Dose }(\mu \mathrm{g} / \mathrm{mL}) \\
\text { E. coli }\end{array}$ & $\begin{array}{l}\text { Dose }(\mu \mathrm{g} / \mathrm{mL}) \\
\text { E. coli } \mathrm{LB} \text { no salt }\end{array}$ \\
\hline Ampicillin & AMP & Cell wall synthesis inhibitor & 1.2 & 1.4 \\
\hline Cefoxitin & FOX & Cell wall synthesis inhibitor & 1.2 & 1.2 \\
\hline Levofloxacin & LVX & $\begin{array}{l}\text { Fluoroquinolone, DNA gyrase } \\
\text { inhibitor }\end{array}$ & 0.01 & 0.015 \\
\hline Ciprofloxacin & CPR & $\begin{array}{l}\text { Fluoroquinolone, DNA gyrase } \\
\text { inhibitor }\end{array}$ & 0.005 & 0.005 \\
\hline Nitrofurantoin & NTR & $\begin{array}{l}\text { DNA damaging, multiple } \\
\text { mechanisms }\end{array}$ & 2 & 1.6 \\
\hline Trimethoprim & TMP & Folic acid synthesis inhibitor & 0.1 & 0.17 \\
\hline Tobramycin & TOB & Aminoglycoside & 1.5 & 0.45 \\
\hline Gentamycin & GEN & Aminoglycoside & 1 & 0.2 \\
\hline Streptomycin & STR & Aminoglycoside & 2 & 0.6 \\
\hline Clindamycin & CLI & Protein synthesis inhibitor, $50 \mathrm{~S}$ & 40 & 25 \\
\hline Erythromycin & ERY & Protein synthesis inhibitor, $50 \mathrm{~S}$ & 50 & 20 \\
\hline Tetracycline & TET & Protein synthesis inhibitor, $30 \mathrm{~S}$ & 0.25 & 0.25 \\
\hline
\end{tabular}

yeast extract, and $10 \mathrm{~g} / \mathrm{L} \mathrm{NaCl}$ ) and grown overnight followed by resuspension in $25 \%$ glycerol, then aliquoted into $50 \mu \mathrm{L}$ and frozen at $-80^{\circ} \mathrm{C}$. Cultures used for daily experiments were started by adding $20 \mu \mathrm{L}$ of thawed aliquots into $2 \mathrm{~mL}$ of LB media. The culture was incubated at $37^{\circ} \mathrm{C}$ until it reached exponential growth phase and diluted to maintain $10^{4}$ cells per experimental condition.

\section{Compounds and materials}

A total of 12 antibiotics were included in the study as representatives of all major drug classes. Gentamycin (GEN), levofloxacin (LVX), tetracycline (TET), tobramycin (TOB), erythromycin (ERY), ampicillin (AMP), clindamycin (CLI), streptomycin (STR), nitrofurantoin (NTR), cefoxitin (FOX), and trimethoprim (TMP), all from Sigma (St. Louis, MO); and ciprofloxacin (CPR) from MP Biomedicals (Santa Ana, CA). Stock solution at $20 \mathrm{mg} / \mathrm{mL}$ of each antibiotic was stored in $50 \mu \mathrm{L}$ aliquot at $-20^{\circ} \mathrm{C}$ and each aliquot was only frozen and thawed once to preserve potency.

\section{Growth experiment}

Drug concentrations were selected to partially inhibit bacterial growth (10-50\% inhibition) that were first determined by a 12-step concentration series of twofold at each step in 96-well plates (Costar). A $5 \mathrm{~mL}$ stock solution of each drug in LB media was made at 10-fold of their respective concentrations (Table 1). For drug pair experiments, $10 \mu \mathrm{L}$ of each component drug was mixed into 96-well plates followed by the addition of $80 \mu \mathrm{L}$ cell inoculum; while $10 \mu \mathrm{L}$ of LB media was added in replacement of a second drug for single-drug experiments.
Replicate plates were prepared from the same antibiotic stock solution to minimize variation and incubated at $300 \mathrm{r}$. p.m. in parallel at various temperatures $\left(22^{\circ} \mathrm{C}, 25^{\circ} \mathrm{C}\right.$, $\left.30{ }^{\circ} \mathrm{C}, 37^{\circ} \mathrm{C}, 41^{\circ} \mathrm{C}, 44^{\circ} \mathrm{C}, 46^{\circ} \mathrm{C}\right)$. OD600 measurements for cell density were taken after 4-h, 8-h, 12-h, and 24-h growth. To examine the drug interaction clustering of $E$. coli in a different external environment, we used LB media without salt, prepared with $10 \mathrm{~g} / \mathrm{L}$ tryptone, $5 \mathrm{~g} / \mathrm{L}$ yeast extract.

\section{Relative growth and interactions}

The relative growth under stressor $x$ (presence of a drug or a non-optimal temperature) is defined as $w_{x}=\frac{g_{x}}{g_{\varphi}}$, where $g_{x}$ is the growth of the bacterial culture under stressor $x$ and $g_{\varphi}$ is the growth of the culture at reference state $\varphi$-the culture at its optimal temperature for growth in the absence of antibiotics $\left(41{ }^{\circ} \mathrm{C}\right.$ in our study). Under the Bliss Independence criterion [38], an interaction is additive if $w_{x y}=w_{x} w_{y}$, where $w_{x y}=\frac{g_{x y}}{g_{\varphi}}$ is the relative growth when stressors $x$ and $y$ are both present. The deviation from additivity is defined as $\varepsilon_{x y}=w_{x y}-w_{x} w_{y}$. An interaction exists when this measure is significantly nonzero. The sign of this measure determines the interaction type $\left(\varepsilon_{x y}<0\right.$ corresponds to synergy and $\varepsilon_{x y}>0$ to antagonism). The raw $\varepsilon_{x y}$ is then rescaled by appropriate reference values as in (Yeh et al. [37]) to yield a rescaled measure, $\tilde{\varepsilon}_{x y}$. Its magnitude can be interpreted as the strength of interaction (see Supplemental Information for details).

\section{Statistics of interaction effects}

With the experimental procedure detailed above, four measurements of the OD600 were taken at every time point, 
each one corresponding to a different experimental replicate, for each stressor, and all possible pairwise combinations. The OD (optical density) values were used as a proxy that is proportional to the absolute growth $\left(g_{x}, g_{y}, g_{x y}, g_{\varphi}\right)$. We chose the point estimate $\hat{g}_{x}$ to be the sample mean of the four measurements of absolute growth under condition $x$. The point estimate for each relative growth was taken to be $\hat{w}_{x}=\frac{\hat{g}_{x}}{\hat{g}_{0}}$ and the point estimate for the interaction $\widehat{\tilde{\varepsilon}}_{x y}=$ $\tilde{\varepsilon}\left(\hat{w}_{x}, \hat{w}_{y}, \hat{w}_{x y}\right)$ (where $\tilde{\varepsilon}$ refers to the rescaled interaction as a function of the relative growths). We followed a parametric bootstrap approach for constructing a 95\% confidence interval $\left(\tilde{\varepsilon}_{2.5}, \tilde{\varepsilon}_{97.5}\right)$ for each interaction, with the assumption that the OD measurements under each condition follow a log-normal distribution.

\section{Network of antibiotic and temperature effects and monochromatic clustering}

We constructed an interaction network where nodes represent the stressors (i.e., drugs or temperatures), and colored edges represent non-additive interactions, with the edge color corresponding to interaction type (red for synergy and green for antagonism). The discretized interaction (i.e., color) of an edge is defined to be

$$
c\left(\tilde{\varepsilon}_{2.5}, \tilde{\varepsilon}_{97.5}\right)=\left\{\begin{array}{ccc}
1 & (\text { green/antagonism }) & \tilde{\varepsilon}_{2.5}>\tilde{\varepsilon}_{r e f} \\
-1 & \text { (red/synergy) } & \tilde{\varepsilon}_{97.5}<-\tilde{\varepsilon}_{r e f}, \\
0 & \text { (no edge) } & \text { otherwise }
\end{array}\right.
$$

where $\tilde{\varepsilon}_{\text {ref }}=0.2$ represents the limit for an interaction to be considered approximately additive. The stressors in the interaction network were clustered into monochromatic classes using a newly developed modification of the Prism 2 algorithm [37]. For details about the clustering algorithm, see the Supplemental Information.

\section{Gene expression profile}

We measured expression of about 1800 genes in E. coli using a library with transcriptional fusions of GFP to each promoter [40]. Strains were maintained in $15 \%$ glycerol at $-80^{\circ} \mathrm{C}$ before inoculating and grown overnight in LB medium with $25 \mu \mathrm{g} / \mathrm{mL}$ of kanamycin in 384 well plates. Cultures were then transferred and pinned into $50 \mu \mathrm{L}$ of LB medium per well, followed by a 4-h incubation at $37^{\circ} \mathrm{C}$ to allow growth up to exponential phase. To measure differential expression at high temperature, cultures were moved to $44{ }^{\circ} \mathrm{C}$ where OD at $595 \mathrm{~nm}$ and GFP fluorescence (excitation, $480 \mathrm{~nm}$; emission, $535 \mathrm{~nm}$ ) were measured every $2 \mathrm{~h}$ for $20 \mathrm{~h}$ using a programmable robotic system (Thermo Cytomat). For expression profile with antibiotic treatment, cultures were pinned into $30 \mu \mathrm{L}$ of $\mathrm{LB}$ medium before 4-h incubation, and another $30 \mu \mathrm{L}$ of $\mathrm{LB}$ medium with the corresponding antibiotic was added into the plates (final concentration: STR at $4 \mu \mathrm{g} / \mathrm{mL}$ and TET at $1 \mu \mathrm{g} / \mathrm{mL}$ ). Controls were carried out at $37^{\circ} \mathrm{C}$ without temperature shift or addition of antibiotics. Antibiotics and control conditions were measured using the same robotic system and timeframe.

\section{Determining over- and under-expressed promoters}

The raw OD and GFP fluorescence measurements for each strain in the promoter library were backgroundcorrected and normalized to yield a GFP/OD value that is proportional to the total GFP fluorescence per cell. The median-normalized GFP/OD values were used to calculate $\log 2$-fold changes in promoter expression for each experimental condition $\left(44^{\circ} \mathrm{C}\right.$, STR, TET) with respect to the control condition $\left(37^{\circ} \mathrm{C}\right)$. Four replicates were averaged to yield a final value of the $\log 2$-fold change $x_{c p t}$ in gene expression (for each promoter $p$ in experimental condition $c$ at time $t$ ). For more details, see Supplemental Information.

As a measure of overall similarity between the gene expression profiles of experimental conditions $c_{1}$ and $c_{2}$, we calculated the mean absolute distance

$d_{c_{1}, c_{2}}=\frac{1}{P T} \sum_{p, t}\left|x_{c_{1} p t}-x_{c_{2} p t}\right|$

of the respective $\log 2$-fold changes, where $P$ and $T$ are the total number of promoters in the library and measured time points, respectively.

For gene ontology (GO) term analysis, we determined over-expressed (OE) and under-expressed (UE) promoters as compared to control with the robust $z$-score method, as in ref. [41]. Details are available in the Supplemental Information. The strains in the promoter library were mapped to GO terms. GO terms of the "biological process" category that are over-represented in the $\mathrm{OE}$ and UE sets for each condition were found by ranking the terms using the $p$-value from Fisher's exact test.

\section{Drug sensitivity profile for heat-adapted strains}

We profiled the antibiotic sensitivity of 10 hightemperature-adapted E. coli strains collected and described by Rodríguez-Verdugo et al. [33]. In addition, we compared their sensitivity profiles with their ancestor strain $(E$. coli B genotype REL1206). Growth after 24-h was measured through OD for each strain exposed to each of 12 drugs under an 11-step concentration series with twofold increase per step. Three replicates of these measurements were obtained using the same methodology as described 


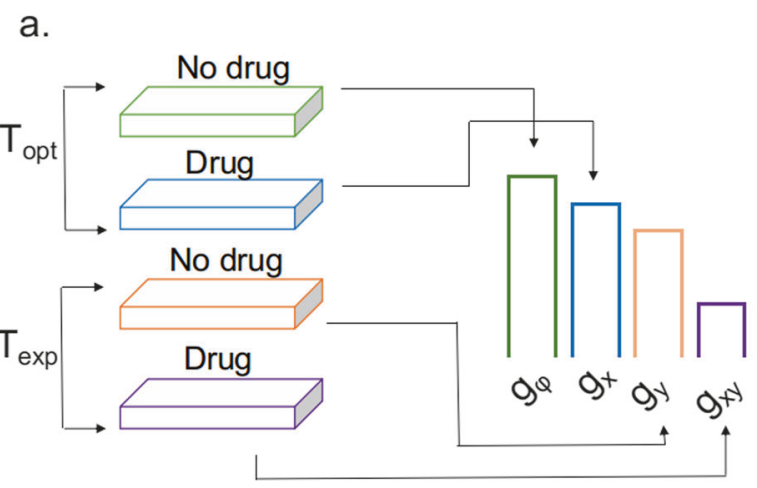

Fig. 1 Schematic illustration of the approach taken in this work. a Growth is measured in the following conditions: reference growth $g_{\varphi}$ at the optimal temperature $\left(T_{\mathrm{opt}}=41{ }^{\circ} \mathrm{C}\right)$ in the absence of drug, $g_{x}$ at optimal temperature with drug, $g_{y}$ at experimental temperature $T_{\text {exp }}$, but no drug, and $g_{x y}$ at non-optimal temperature with drug. b The growth of each experimental condition is converted to proportions $w_{x}$, $w_{y}, w_{x y}$ by dividing by the reference growth. The difference between $w_{x y}$ (observed growth) and the product of $w_{x}$ and $w_{y}$ (expected growth under independence) is then used to classify the interaction between

in the Growth experiment section. IC50 values and their associated credible intervals were determined by fitting a five-parameter logistic model to the growth curve for each strain. Details about the model and fitting can be found in the Supplemental Information.

\section{Results}

\section{Clustering of an antibiotic and temperature stressor interaction network reveals overlap in their physiological effects}

To find groups of stressors (i.e., antibiotics and/or temperatures) that have similar effects on E. coli physiology, we first evaluate the interactions - synergy, additivity, or antagonism -between each pair of antibiotics, and between each antibiotic and a range of growth temperatures $\left(22^{\circ} \mathrm{C}, 25^{\circ} \mathrm{C}\right.$, $30{ }^{\circ} \mathrm{C}, 37^{\circ} \mathrm{C}, 44^{\circ} \mathrm{C}, 46{ }^{\circ} \mathrm{C}$ ) (Fig. 1a, b). The maximum growth in the absence of antibiotic was observed at $41^{\circ} \mathrm{C}$ : this optimum growth temperature was chosen as the unstressed reference state for evaluating the relative growth in the presence of each stressor. We evaluate the interactions based on the 24-h growth of E. coli after exposure to the corresponding stressors. We then construct a SIN (Fig. 1c) where nodes represent the stressors and colored edges represent interaction type (discretized based on a hypothesis test, see Fig. 1c and Methods). The resulting network is clustered to find monochromatically interacting groups, which correspond to similar interaction profiles, using our b.

$w_{x}=\frac{g_{x}}{g_{\phi}} \quad w_{y}=\frac{g_{y}}{g_{\phi}} \quad w_{x y}=\frac{g_{x y}}{g_{\phi}}$

C.

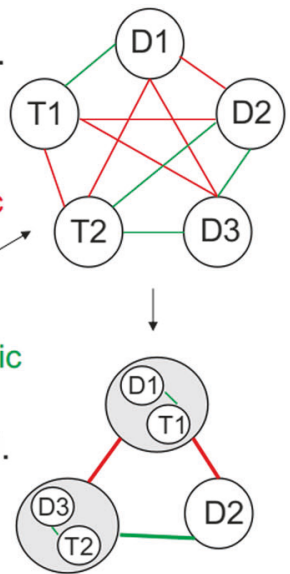

drugs and temperatures into three cases: synergistic (red line), additive (white or not shown), and antagonistic (green line), $\mathbf{c}$ which can be represented as an interaction network. d Drugs and temperatures can then be clustered into a functional class based on the monochromaticity of interactions with a different class. This example shows a drug-temperature interaction, but drug-drug interactions are obtained similarly, by replacing the growth with no drug at $T_{\exp }$ with the growth under a second drug at $T_{\mathrm{opt}}$

modified Prism 2 algorithm (Fig. 1d, see Supplemental Information for details of the algorithm). These groups consist of drugs/temperatures that have similar overall interactions with other stressors, regardless of their interaction type. Consistent with overlap in the mechanism of action of specific drugs and the physiological effect of non-optimal temperatures, we find that the evaluated temperatures cluster with antibiotics in the following three groups (Fig. 2): (1) all temperatures lower than the temperature for peak growth cluster together, along with the fluoroquinolones (LVX, CPR), which are DNA gyrase inhibitors, and with the $30 \mathrm{~S}$ protein synthesis inhibitor tetracycline (TET); (2) the temperature $44^{\circ} \mathrm{C}$ clusters with the DNA-damaging drug nitrofurantoin (NTR) and with trimethoprim (TMP), an inhibitor of the folic acid biosynthesis pathway that is responsible for generating an essential DNA precursor; (3) the highest evaluated temperature, $46^{\circ} \mathrm{C}$, clusters with the aminoglycosides (GEN, STR, TOB), antibiotics that affect protein translation proofreading [42]. We thus conclude that monochromatic clustering successfully separated the antibiotics according to their mechanism of action and/or grouped with temperatures that have similar physiological effects.

To evaluate the robustness of our clustering results, we also measure interactions in a different condition: LB medium with no salt (Supplemental Figure 6). As before, we find that high temperatures cluster with aminoglycosides (GEN, STR, TOB) and TMP, but now they cluster in a single group. Low temperatures cluster in a single group with CLI, a $50 \mathrm{~S}$ protein synthesis inhibitor, instead of TET, LVX, CPR. 

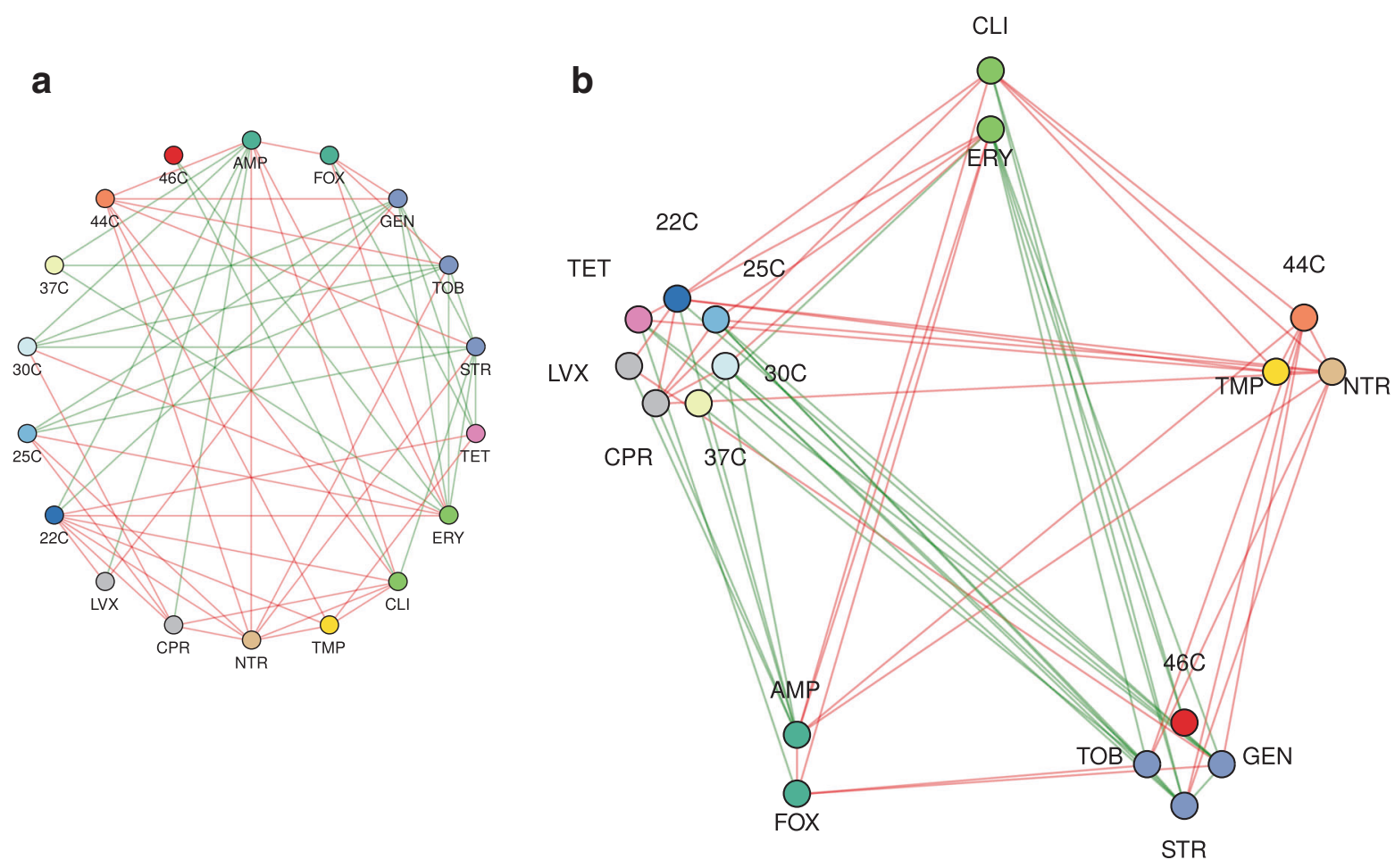

Fig. 2 Monochromatic clustering of the interaction network. a Unclustered interaction network. The nodes that correspond to drugs are color-coded by their mechanism of action (Table 1), and the nodes that correspond to temperatures are colored in a gradient from blue

(low) to red (high). The edges correspond to discretized interaction type, as in Fig. 3c: synergy (red), antagonism (green), additive or unknown (no edge). b Network clustered into monochromatic classes by the modified Prism2 algorithm (Supplemental Information)

\section{Patterns in antibiotic and temperature interactions}

We find that the distribution of the interactions between all pairs of stressors at 24-h growth is trimodal (Fig. 3a), with peaks that correspond to synergy $\left(\tilde{\varepsilon}_{x y} \approx-1\right)$, additivity $\left(\tilde{\varepsilon}_{x y} \approx 0\right)$, and antagonism $\left(\tilde{\varepsilon}_{x y} \approx 1\right)$, similar to previous work [37]. Ampicillin (AMP) and the aminoglycosides (GEN, STR, TOB) are mostly antagonistic with temperatures lower than the optimum $\left(41{ }^{\circ} \mathrm{C}\right)$, and synergistic or additive with higher temperatures (Fig. 3b). Erythromycin and clindamycin (ERY, CLI) exhibit the opposite pattern: they are mostly synergistic with lower temperatures and antagonistic with temperatures near the optimum or higher, with the exception of $44^{\circ} \mathrm{C}$. All interactions were calculated using the mean growth under each condition (see Methods). Using median values yields similar results (Supplemental Fig. 1). The distribution of interactions changes with different choices of growth measurement time points (Supplemental Fig. 2). The 24-h time point was chosen for the analysis since the effects on growth and interactions of many of the stressors are not apparent at earlier time points (Supplemental Information).

\section{Gene expression dynamics after exposure to antibiotics and high temperatures}

Next, we explore the molecular mechanisms involved in the response to antibiotics by evaluating genome-wide transcriptional dynamics after exposure to high temperature $\left(44{ }^{\circ} \mathrm{C}\right)$ and two representative drugs that clustered with temperatures: TET (22-37 ${ }^{\circ} \mathrm{C}$, cold cluster) and STR $\left(46^{\circ} \mathrm{C}\right.$ cluster). To do this, we measure fold changes in gene expression compared to a control condition $\left(37^{\circ} \mathrm{C}\right)$ with a library of E. coli strains containing GFP fused to more than 1800 promoters [40]. Consistent with the drug-temperature clusters, we find that the overall gene expression at $44{ }^{\circ} \mathrm{C}$ is more similar to the response to STR than to TET (Fig. 4a).

We also look at the expression of some canonical genes from the heat-shock and cold-shock responses (Fig. 4b). We find that $\operatorname{csp} A$ and $\operatorname{csp} G$, main regulators of the coldshock response, are over-expressed only in response to TET, while $\operatorname{csp} B$ and $c s p I$ are under-expressed. We find that $r p o H$, the main regulator of the heat-shock response is over-expressed compared to control only in the $44{ }^{\circ} \mathrm{C}$ condition and is under-expressed upon exposure to TET. However, chaperones involved in the heat-shock response 


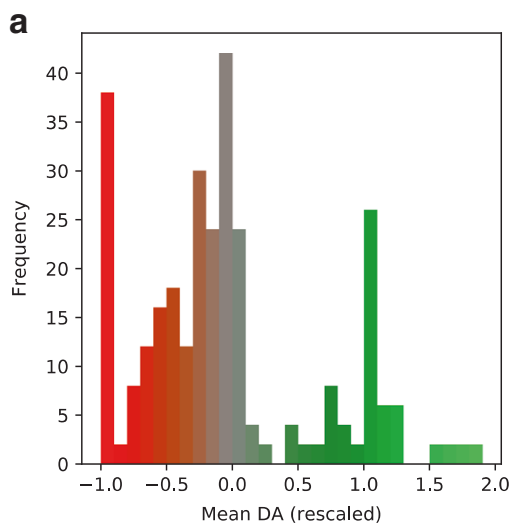

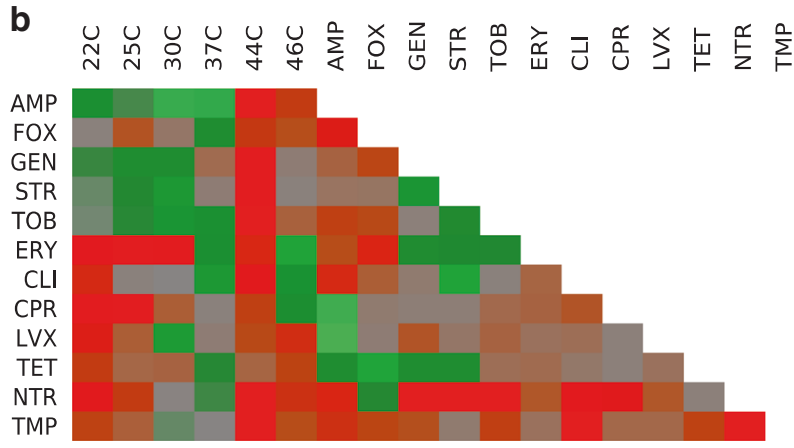

Fig. 3 Interaction effects between antibiotics and temperature based on growth after $24-\mathrm{h}$. The interaction effect $(\tilde{\varepsilon})$ values are color-coded in a gradient, from synergy (red) to additive (gray) and antagonism (green). a Overall distribution of the mean estimated interaction effects across all treatments. The distribution shows three clear peaks, corresponding

(dnaJ, dnaK, groE, grpE) show increased expression under both STR and TET. This indicates that components of the heat-shock response are being activated in response to both antibiotics, while the cold-shock response is activated only for TET (a drug from the low temperature cluster). Analysis of gene ontology terms shows that genes involved in the response to unfolded proteins, which is a well-known effect of high-temperature stress, are induced in response to STR (Fig. 4c).

\section{Changes in antibiotic sensitivity for heat-adapted E. coli strains}

Our above experiments evaluate the overlap between the existing responses of wild-type $E$. coli to antibiotics and temperature. It is also of interest to evaluate if there is crossresistance between temperature-adapted strains and antibiotics. Previously, Rodríguez-Verdugo et al. [33] adapted an E. coli strain for over 2000 generations at $42.2{ }^{\circ} \mathrm{C}$ and showed the heat-adapted strains acquired resistance to rifampicin. The resistance phenotype was mapped to mutations in the $r р о B$ gene [33]. We profile 10 of the heatadapted strains, their ancestor strain, and 3 different $r p o B$ mutants exposed to the antibiotics used in the clustering

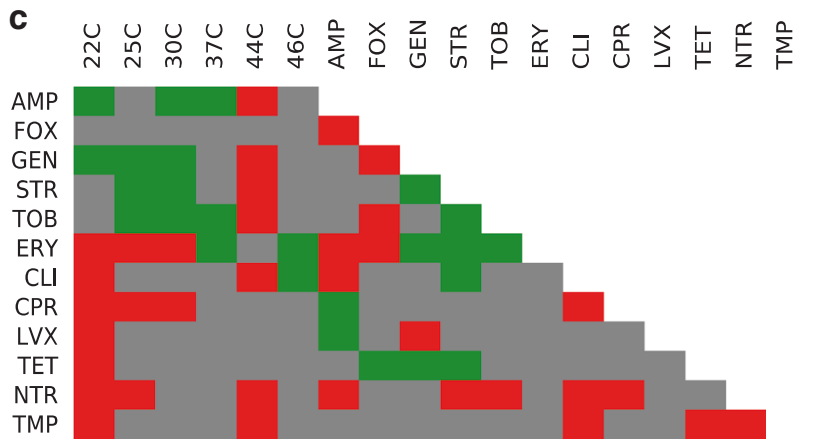

to strong synergy, additivity, and antagonistic buffering. b Matrix heatmap of the mean interaction effects. Antibiotics with the same mechanism of action show similar interaction patterns. c Matrix heatmap of the discretized interaction types used for constructing the edges of the interaction network

experiment. As predicted from our clustering analysis, most heat-adapted strains are as or more resistant to antibiotics (NTR and TMP) that mimic the effects of high temperatures $\left(44^{\circ} \mathrm{C}\right)$ (Fig. 5a, b). Resistance to aminoglycosides (which clustered with $46^{\circ} \mathrm{C}$, a much higher temperature to the one the heat-adapted strains were evolved on) was higher in some temperature-adapted strains and lower in others. Moreover, compared with the ancestor strain, most heatadapted strains are more sensitive to antibiotics that mimic the effects of cold temperatures such as protein synthesis inhibitors (CLI, ERY, TET) (Fig. 5a, b, Supplemental Fig. 5). These results are based on changes in IC50, the antibiotic concentration that results in 50\% growth.

Intriguingly, the same patterns were not observed for the $r p o B$ mutants exposed to some drugs (e.g., for ERY, CLI). These mutants were not adapted at high temperature, suggesting that there are additional adaptive mutations in the heat-adapted strains besides rрoB.

\section{Discussion}

In this paper, we cluster interactions among drugs and temperatures to infer that there are shared physiological 
a

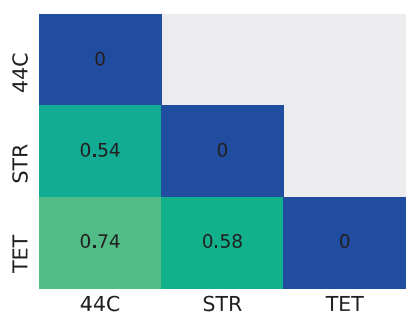

heat

b
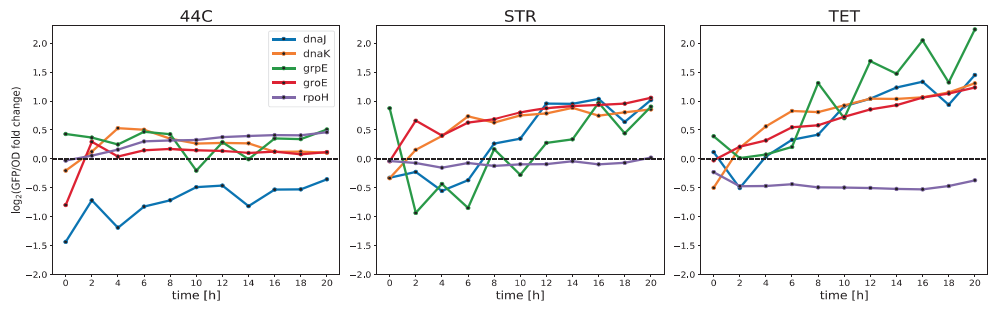

cold
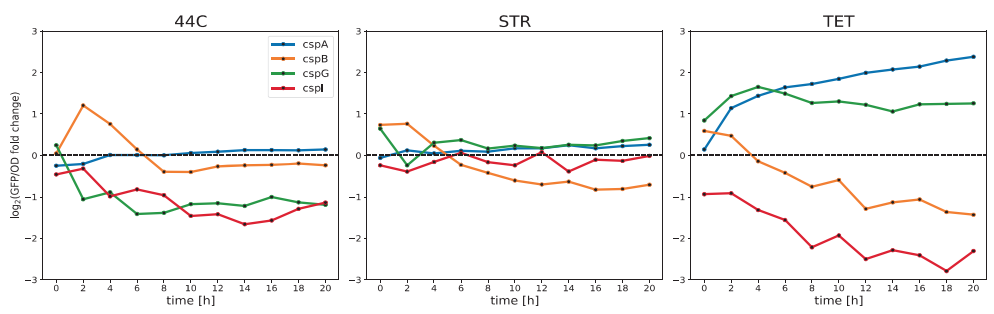

C

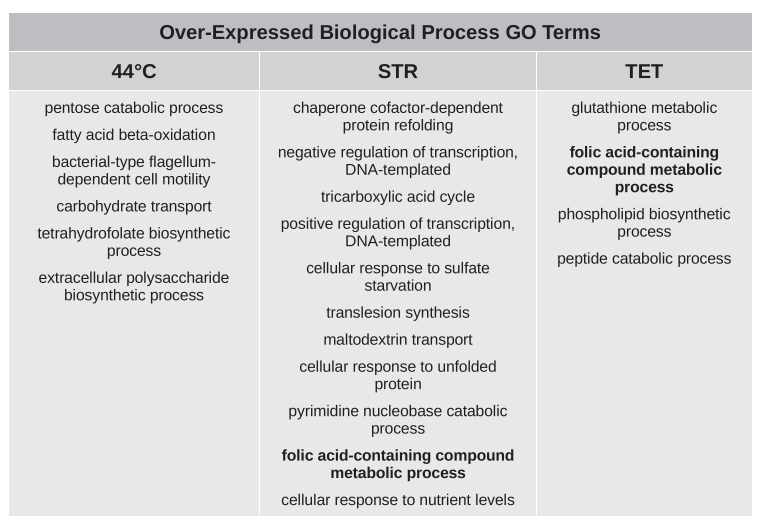

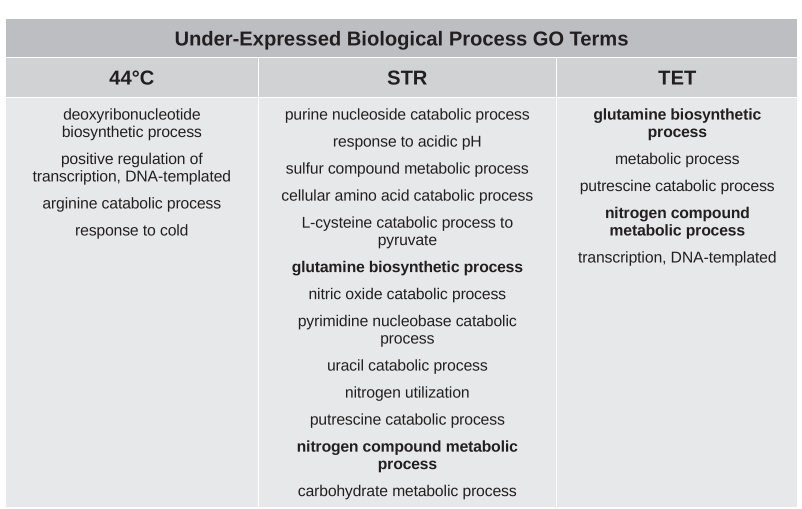

Fig. 4 Gene expression of E. coli after exposure to antibiotics and high temperature. The gene expression response of $E$. coli was evaluated with a library of 1870 fluorescent transcriptional reporters. a Mean absolute gene expression distance between experimental conditions. Lower numbers indicate conditions with more similar gene expression profiles. b Gene expression of representative heat-shock and

responses of E. coli to these stressors. Our SIN analysis suggests that the stress responses to low temperatures overlap with those of antibiotics that affect DNA gyrase and a $30 \mathrm{~S}$ protein synthesis inhibitor. In addition, the stress responses to high temperatures overlap with those of drugs that affect protein translation proofreading and drugs that damage DNA. Due to this overlap, we conclude that cellular responses to temperature stress have likely been evolutionarily co-opted to also respond to many classes of antibiotic stress. Because pressure and $\mathrm{pH}$ are also ancient stressors, we expect that responses to them may have also been coopted to deal with antibiotic stress. Our approach provides a powerful basis for asking similar questions about other environmental, chemical, or physical stressors that affect the population growth of an organism.

We show that monochromatic clustering successfully separates antibiotics and temperatures into groups that have cold-shock genes relative to control $\left(37^{\circ} \mathrm{C}\right)$ in response to experimental conditions $\left(44^{\circ} \mathrm{C}, \mathrm{STR}, \mathrm{TET}\right)$. c Gene ontology terms in the biological process category over-represented in the set of overexpressed and under-expressed genes in each experimental condition relative to control. Terms that are in bold occur in more than one treatment

similar effects on bacterial physiology (Table 2). First, all temperatures $\left(22^{\circ} \mathrm{C}, 25^{\circ} \mathrm{C}, 30^{\circ} \mathrm{C}, 37^{\circ} \mathrm{C}\right)$ lower than the optimum $\left(41^{\circ} \mathrm{C}\right.$, which results in the highest growth) cluster with antibiotics that either affect the early stages of protein synthesis (TET prevents the association of aminoacyl tRNAs with the ribosome [43]) or are DNA gyrase inhibitors (LVX, CPR). This is consistent with the known effects of low temperature. One of the main effects of cold shock is translational block, which is thought to most likely occur at the translation initiation step [44]. Some previous reports have also shown that cold-shock induces the expression of DNA gyrase and a transient increase of negative supercoiling of DNA in E. coli [44-46]. Second, the highest evaluated temperature $\left(46^{\circ} \mathrm{C}\right)$ clusters with the aminoglycosides, antibiotics that affect protein translation proofreading [42]. This leads to misfolding and aggregation of defective proteins that mimic the well-known effects of 
a

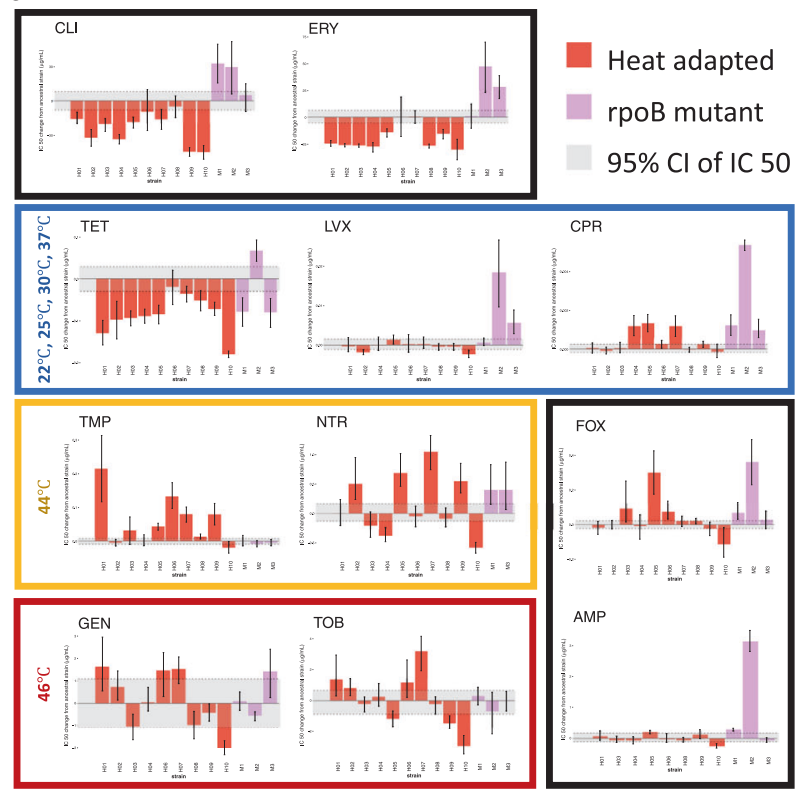

Fig. 5 Antibiotic sensitivity of high-temperature-adapted E. coli strains. a Absolute change in the IC50 $(\mu \mathrm{g} / \mathrm{mL})$ relative to the ancestral strain. Heat-adapted strains (red), rpoB mutant strains (purple). Error bars represent $95 \%$ credible intervals (CIs). Gray region represents the 95\% CI of ancestral strain. Drugs are grouped according to the clusters of antibiotics and temperature in (Fig. 2). Conditions where the model fit was poor were removed from the plots (Supplemental Information). b

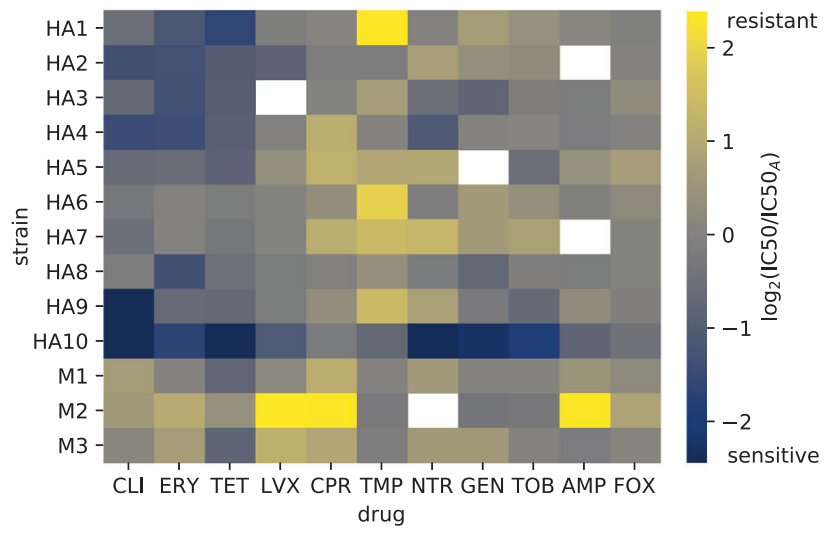

b Heatmap of $\log 2$ fold changes from the ancestral IC50. Heat-adapted strains are denoted by HA, while rpoB mutant strains are denoted by M. Positive numbers (yellow) indicate increased IC50 (more resistance), while negative numbers (blue) indicate a decreased IC50 (higher sensitivity). Drugs are grouped in the same way as in a. Missing conditions are shown in white

Table 2 Effects of temperature and antibiotics in cellular physiology

\begin{tabular}{llll}
\hline & Temperature & & Antibiotics \\
\cline { 2 - 4 } & High & Low & \\
\hline DNA & Decreased negative supercoiling & Increased negative supercoiling & $\begin{array}{l}\text { CPR, LVX: DNA gyrase inhibitors } \\
\text { NTR, TMP: DNA damage }\end{array}$ \\
Protein & Misfolding and aggregation & Translational block & $\begin{array}{l}\text { ERY, CLI, TET: inhibition of translation } \\
\text { GEN, STR, TOB: increased errors in } \\
\text { translation }\end{array}$ \\
& $\begin{array}{l}\text { Increased membrane fluidity, damage } \\
\text { to membrane due to aggregation } \\
\text { of membrane proteins }\end{array}$ & $\begin{array}{l}\text { Decreased membrane fluidity and } \\
\text { altered fatty acid composition }\end{array}$ & $\begin{array}{l}\text { GEN, STR, TOB: damage to membrane } \\
\text { due to incorporation of misfolded } \\
\text { membrane proteins }\end{array}$ \\
\hline
\end{tabular}

high temperatures on protein stability and folding [21]. Finally, $44^{\circ} \mathrm{C}$ clusters separately from $46^{\circ} \mathrm{C}$, with antibiotics that either damage nucleic acids or inhibit their synthesis. This intriguing finding suggests the main physiological effect of this temperature (compared to $41^{\circ} \mathrm{C}$ ) could be due to effects on nucleic acids. This connection to nucleic acids is suggestive given that the heat-shock protein Hsp70 enhances repair of UV-induced DNA damage [47]. We speculate that this specific temperature clustering separately from the aminoglycosides may be due to the heat-shock response being able to partially combat protein unfolding at $44{ }^{\circ} \mathrm{C}$, but not $46^{\circ} \mathrm{C}$.
An important feature of the monochromatic clustering framework is that it implicitly assumes each node (i.e., stressor) in the SIN belongs to a single cluster. This single cluster assumption is likely a good approximation for antibiotics as they tend to bind to specific cellular targets. However, physical or environmental stressors, such as temperature, can affect many cellular processes simultaneously. Because of this, the clusters in our study correspond to consensus effects: these are informative summaries of the dominant effects of the environmental stressor, but could miss secondary effects that are not shared with the other members of the cluster. Further theoretical and 
computational work could focus on relaxing the single cluster assumption of monochromatic clustering to allow temperatures to be grouped with multiple, potentially dissimilar classes of antibiotics. This updated methodology could allow a more nuanced approach, capable of breaking down the effects of an environmental stressor in terms of more targeted perturbations such as antibiotics, chemical inhibitors, or gene deletions that are deleterious to different cellular subsystems.

Indeed, some of the evaluated temperatures do have similar interactions to antibiotics in different clusters (Supplemental Fig. 5). Examples are the lowest temperatures evaluated $\left(22^{\circ} \mathrm{C}, 25^{\circ} \mathrm{C}\right)$. These temperatures have similar interaction profiles to both the 30S (TET) and 50S (ERY, CLI) protein synthesis inhibitors, while higher temperatures that are still below the optimum $\left(30^{\circ} \mathrm{C}, 37^{\circ} \mathrm{C}\right)$ are only similar to the $30 \mathrm{~S}$ inhibitors. This is reflected in (ERY, CLI) being in a separate cluster from the low temperatures. Interestingly, low temperatures cluster with CLI under no salt conditions (Supplemental Figure 6), and a previous report has shown cold-shock proteins are induced in response to CLI [48].

Clinically, the impact of temperature on the effects of antibiotics is also of interest because it suggests some antibiotics could have increased or reduced effectiveness in patients with fever or hypothermia. Previous work has shown there is increased resistance to gentamicin (GEN) in Francisella tularensis, Listeria monocytogenes, and Klebsiella pneumoniae at $26^{\circ} \mathrm{C}$ when compared to $37^{\circ} \mathrm{C}$ [32]. This increased resistance seems to be mediated by reduced drug uptake. It has also been reported that streptomycin (STR), tetracycline (TET), ampicillin (AMP), and cefoxitin (FOX) have increased effectiveness at $46^{\circ} \mathrm{C}$ compared to $37^{\circ} \mathrm{C}$ in Pseudomonas aeuriginosa [49]. Our results are consistent with both reports, as we found: (1) aminoglycosides (GEN, STR, TOB) are mostly synergistic with high temperatures and antagonistic with low temperatures, (2) synergy of beta-lactams (AMP and FOX) with high temperatures, and (3) synergy of TET with $46^{\circ} \mathrm{C}$ (but, interestingly, with $22^{\circ} \mathrm{C}$ as well). Some other antibiotics (LVX, NTR, and TMP) also exhibit this curious pattern of being synergistic with both temperature extremes and either additive or slightly antagonistic with less stressful temperatures. Further work is needed to obtain a more detailed understanding of these interaction patterns.

Our transcriptional analysis shows that the overall expression patterns of E. coli exposed to high temperature are more similar to those induced by STR than those induced by TET (Fig. 4a). We find that $c s p A$ and $c s p G$, main cold-shock response regulators in E. coli, have increased expression in response to TET, but not STR or high temperature (Fig. 4b). Other cold-shock regulators $(\operatorname{csp} B, \operatorname{csp} I)$ show decreased expression. It has been shown that cold-shock genes are differentially induced depending on the severity of the cold stress. In particular, $\operatorname{csp} A$ expression is induced between $20-30{ }^{\circ} \mathrm{C}$, while cspI is induced between 10 and $15^{\circ} \mathrm{C}$ [50]. These gene expression results are in agreement with our drug/temperature clusters, since the low temperature cluster contains temperatures between 22 and $37^{\circ} \mathrm{C}$. We find that genes involved in the response to unfolded protein (as determined by gene ontology annotations), which commonly results from heat stress, are overrepresented in the genes induced by STR, a representative antibiotic that clustered with heat (Fig. 4c). Interestingly, some heat-shock response genes that combat unfolded protein stress (dnaK, dnaJ, groE, grpE) have increased expression in response to both STR and TET. However, the main heat-shock response regulator $r p o H$ is not over-expressed in response to either antibiotic (in fact, is under-expressed in response to TET). Together, these results suggest heat-shock genes participate in the response to both antibiotics. However, they may be activated in a different way than the canonical heat-shock response.

Our clustering and gene expression results show that multiple antibiotics (particularly aminoglycosides, TET, DNA gyrase inhibitors, and DNA-damaging antibiotics) have similar overall effects in $E$. coli physiology to specific low or high temperatures. This is consistent with components of the stress response to temperature having been coopted over evolutionary time to deal with antibiotics that disrupt similar cellular structures or functions to those affected by low- and high-temperature stress. Moreover, we show that this overlap between stress responses can be related to the acquired cross-resistance of temperatureadapted strains to specific groups of antibiotics.

Typically, the overlap between cellular responses to stress has been studied by isolating subcellular parts and attempting to piece together this information to understand stress responses at the whole-cell level. Here, we take a reverse, yet complementary approach: by studying the effect of perturbations on the whole system, we gain more insights into the mechanisms of its specific parts. Importantly, no aspect of this methodology is specific to antibiotics and temperature. Our SIN clustering method can be used to evaluate shared responses among any combination of physical, chemical, and/or biological stressors that affect organismic growth.

In conclusion, we evaluate if the overlap between antibiotic and temperature stress responses is predictive of the cross-resistance of high-temperature-adapted strains to antibiotics (Fig. 5). We find that high-temperature-adapted strains become more sensitive to protein synthesis inhibitors (CLI, ERY, TET), drugs that either clustered with or are similar to low temperatures. In contrast, the temperatureadapted strains become more resistant to drugs that clustered with $44{ }^{\circ} \mathrm{C}$ (NTR, TMP), but not necessarily to drugs 
that cluster with $46^{\circ} \mathrm{C}$ (GEN, STR, TOB). Overall, these results strongly suggest that seemingly novel drug resistance is conferred to strains via adaptations they acquired while being evolved at extreme temperatures. Specifically, strains adapted to heat $\left(42.2^{\circ} \mathrm{C}\right)$ are more resistant to drugs that damage DNA (which cluster with $44{ }^{\circ} \mathrm{C}$, a similar temperature), while also being more sensitive to drugs that mimic the effect of cold. However, this pattern is not universal, since the strains do not become more sensitive to fluoroquinolones (LVX, CPR), which also cluster with cold. Interestingly, the rроB mutants do not follow the same antibiotic resistance patterns as the temperatureadapted strains, suggesting there may be more adaptive mutations to temperature besides rрoB.

Acknowledgements We are grateful for funding from the Hellman Foundation (P.J.Y.), a KL2 Fellowship (P.J.Y.) through the NIH/ National Center for Advancing Translational Science (NCATS) UCLA CTSI Grant Number UL1TR001881, and a James F. McDonnell Foundation Complex Systems Scholar Award (V.M.S.). Author M.C.-L. is supported by UC-MEXUS and CONACYT. The hightemperature-adapted strains used in this paper were kindly donated by Alejandra Rodríguez-Verdugo, Brandon Gaut, and collaborators (Rodríguez-Verdugo et al., 2013).

Disclaimer This investigation was supported by NIH, under Ruth L. Kirschstein National Research Service Award (T32-GM008185). Its contents are solely the responsibility of the authors and do not necessarily represent the official views of the NIH.

Author contributions Conceptualization: M.C.-L., T.M.K., V.M.S. and P.J.Y.; experimental work: T.M.K., R.W., N.A.L. and R.D.; computational work and data analysis: M.C.-L.; theoretical development: M.C.-L., E.T., V.M.S. and P.J.Y; writing: M.C.-L., T.M.K., N.A.L., V.M.S. and P.J.Y.; supervision: V.M.S. and P.J.Y.

\section{Compliance with ethical standards}

Conflict of interest The authors declare that they have no conflict of interest.

\section{References}

1. Hoffmann AA, Parsons PA. Evolutionary genetics and environmental stress. Oxford, England, UK: Oxford University Press; 1991.

2. Meyers LA, Bull JJ. Fighting change with change: adaptive variation in an uncertain world. Trends Ecol Evol. 2002;17: 551-7.

3. Cho H, Uehara T, Bernhardt TG. Beta-lactam antibiotics induce a lethal malfunctioning of the bacterial cell wall synthesis machinery. Cell. 2014;159:1300-11.

4. Kohanski MA, Dwyer DJ, Hayete B, Lawrence CA, Collins JJ. A common mechanism of cellular death induced by bactericidal antibiotics. Cell. 2007;130:797-810.

5. Gilchrist GW. Specialists and generalists in changing environments. I. Fitness landscapes of thermal sensitivity. Am Nat. 1995;146:252-70.

6. Csonka LN, Hanson AD. Prokaryotic osmoregulation: genetics and physiology. Annu Rev Microbiol. 1991;45:569-606.
7. Sharma UK, Chatterji D. Transcriptional switching in Escherichia coli during stress and starvation by modulation of $\sigma 70$ activity. FEMS Microbiol Rev. 2010;34:646-57.

8. Levy SB. Active efflux mechanisms for antimicrobial resistance. Antimicrob Agents Chemother. 1992;36:695.

9. Paulsen IT, Park JH, Choi PS, Saier MH Jr. A family of gramnegative bacterial outer membrane factors that function in the export of proteins, carbohydrates, drugs and heavy metals from gram-negative bacteria. FEMS Microbiol Lett. 1997;156:1-8.

10. Begic S, Worobec EA. Regulation of Serratia marcescens ompF and ompC porin genes in response to osmotic stress, salicylate, temperature and pH. Microbiology. 2006;152:485-91.

11. Kaprelyants AS, Kell DB. Dormancy in stationary-phase cultures of Micrococcus luteus: flow cytometric analysis of starvation and resuscitation. Appl Environ Microbiol. 1993;59:3187-96.

12. Bada JL, Lazcano A. Some like it hot, but not the first biomolecules. Science. 2002;296:1982-3.

13. Braun D, Libchaber A. Thermal force approach to molecular evolution. Phys Biol. 2004;1:P1.

14. Daniel I, Oger P, Winter R. Origins of life and biochemistry under high-pressure conditions. Chem Soc Rev. 2006;35:858-75.

15. Hazen RM, Boctor N, Brandes JA, Cody GD, Hemley RJ, Sharma A, et al. High pressure and the origin of life. J Phys Condens Matter. 2002;14:11489.

16. Schwartzman DW, Lineweaver $\mathrm{CH}$. The hyperthermophilic origin of life revisited. Biochemical Society Transactions. 2004;32 (2):168-71.

17. Stetter KO. Hyperthermophiles in the history of life. Philos Trans R Soc Lond B Biol Sci. 2006;361:1837-43.

18. Åkerfelt M, Morimoto RI, Sistonen L. Heat shock factors: integrators of cell stress, development and lifespan. Nat Rev Mol Cell Biol. 2010;11:545.

19. De AM. Heat shock proteins: facts, thoughts, and dreams. Shock. 1999;11:1-12.

20. Lindquist S. The heat-shock response. Annu Rev Biochem. 1986;55:1151-91.

21. Richter K, Haslbeck M, Buchner J. The heat shock response: life on the verge of death. Mol Cell. 2010;40:253-66.

22. D'Costa VM, King CE, Kalan L, Morar M, Sung WW, Schwarz C, et al. Antibiotic resistance is ancient. Nature. 2011;477:457.

23. Mondal S, Pathak BK, Ray S, Barat C. Impact of P-Site tRNA and antibiotics on ribosome mediated protein folding: studies using the Escherichia coli ribosome. PLoS ONE. 2014;9:e101293.

24. Vabulas RM, Raychaudhuri S, Hayer-Hartl M, Hartl FU. Protein folding in the cytoplasm and the heat shock response. Cold Spring Harb Perspect Biol. 2010;2:a004390.

25. Dragosits M, Mozhayskiy V, Quinones-Soto S, Park J, Tagkopoulos I. Evolutionary potential, cross-stress behavior and the genetic basis of acquired stress resistance in Escherichia coli. Mol Syst Biol. 2013;9:643.

26. Święciło A. Cross-stress resistance in Saccharomyces cerevisiae yeast-new insight into an old phenomenon. Cell Stress Chaperones. 2016;21:187-200.

27. Völker U, Mach H, Schmid R, Hecker M. Stress proteins and cross-protection by heat shock and salt stress in Bacillus subtilis. Microbiology. 1992;138:2125-35.

28. Battesti A, Majdalani N, Gottesman S. The RpoS-mediated general stress response in Escherichia coli. Annu Rev Microbiol. 2011;65:189-213.

29. Gruber TM, Gross CA. Multiple sigma subunits and the partitioning of bacterial transcription space. Annu Rev Microbiol. 2003;57:441-66.

30. Somorin Y, Abram F, Brennan F, O'Byrne C. The general stress response is conserved in long-term soil-persistent strains of Escherichia coli. Appl Environ Microbiol. 2016;82:4628-40. 
31. Cardoso K, Gandra RF, Wisniewski ES, Osaku CA, Kadowaki MK, Felipach-Neto V, et al. DnaK and GroEL are induced in response to antibiotic and heat shock in Acinetobacter baumannii. J Med Microbiol. 2010;59:1061-8.

32. Loughman K, Hall J, Knowlton S, Sindeldecker D, Gilson T, Schmitt DM, et al. Temperature-dependent gentamicin resistance of Francisella tularensis is mediated by uptake modulation. Front Microbiol. 2016;7:37.

33. Rodríguez-Verdugo A, Gaut BS, Tenaillon O. Evolution of Escherichia coli rifampicin resistance in an antibiotic-free environment during thermal stress. BMC Evol Biol. 2013;13:50.

34. Goltermann L, Good L, Bentin T. Chaperonins fight aminoglycoside-induced protein misfolding and promote shortterm tolerance in Escherichia coli. J Biol Chem. 2013;288: 10483-9.

35. Poole K. Stress responses as determinants of antimicrobial resistance in Gram-negative bacteria. Trends Microbiol. 2012; 20:227-34.

36. Segre D, DeLuna A, Church GM, Kishony R. Modular epistasis in yeast metabolism. Nat Genet. 2005;37:77.

37. Yeh P, Tschumi AI, Kishony R. Functional classification of drugs by properties of their pairwise interactions. Nat Genet. 2006;38:489.

38. Bliss C. The toxicity of poisons applied jointly. Ann Appl Biol. 1939;26:585-615.

39. Datsenko KA, Wanner BL. One-step inactivation of chromosomal genes in Escherichia coli K-12 using PCR products. Proc Natl Acad Sci USA. 2000;97:6640-5.

40. Zaslaver A, Bren A, Ronen M, Itzkovitz S, Kikoin I, Shavit S, et al. A comprehensive library of fluorescent transcriptional reporters for Escherichia coli. Nat Methods. 2006;3:623.
41. Reyes VC, Li M, Hoek EM, Mahendra S, Damoiseaux R. Genome-wide assessment in Escherichia coli reveals timedependent nanotoxicity paradigms. ACS Nano. 2012;6:9402-15.

42. Mingeot-Leclercq M-P, Glupczynski Y, Tulkens PM. Aminoglycosides: activity and resistance. Antimicrob Agents Chemother. 1999;43:727-37.

43. Chopra I, Roberts M. Tetracycline antibiotics: mode of action, applications, molecular biology, and epidemiology of bacterial resistance. Microbiol Mol Biol Rev. 2001;65:232-60.

44. Yamanaka K. Cold shock response in Escherichia coli. J Mol Microbiol Biotechnol. 1999;1:193-202.

45. Goldstein E, Drlica K. Regulation of bacterial DNA supercoiling: plasmid linking numbers vary with growth temperature. Proc Natl Acad Sci USA. 1984;81:4046-50.

46. Mizushima T, Kataoka K, Ogata Y, Inoue R, Sekimizu K. Increase in negative supercoiling of plasmid DNA in Escherichia coli exposed to cold shock. Mol Microbiol. 1997;23:381-6.

47. Niu P, Liu L, Gong Z, Tan H, Wang F, Yuan J, et al. Overexpressed heat shock protein 70 protects cells against DNA damage caused by ultraviolet $\mathrm{C}$ in a dose-dependent manner. Cell Stress Chaperones. 2006;11:162-9.

48. VanBogelen RA, Neidhardt FC. Ribosomes as sensors of heat and cold shock in Escherichia coli. Proc Natl Acad Sci USA. 1990;87:5589-93.

49. Bhatti A, Kumar K, Stobo C, Chaudhry G, Ingram J. High temperature induced antibiotic sensitivity changes in Pseudomonas aeruginosa. Microbios. 1999;97:103-15.

50. Wang N, Yamanaka K, Inouye M. CspI, the ninth member of the CspA family of Escherichia coli, is induced upon cold shock. J Bacteriol. 1999;181:1603-9. 